The AstrophysiCal Journal, 369:L41-L44, 1991 March 10

(C) 1991. The American Astronomical Society. All rights reserved. Printed in U.S.A.

\title{
THE CORE OF THE NEARBY S0 GALAXY NGC 7457 IMAGED WITH THE $H S T$ PLANETARY CAMERA ${ }^{1}$
}

\author{
Tod R. Lauer, ${ }^{2}$ S. M. Faber,${ }^{3}$ Jon A. Holtzman, ${ }^{4}$ William A. Baum, ${ }^{5}$ Douglas G. Currie, ${ }^{6}$ S. P. Ewald, ${ }^{7}$ \\ Edward J. Groth, ${ }^{8}$ J. JefF Hester, ${ }^{9}$ T. Kelsall, ${ }^{10}$ Jerome Kristian, ${ }^{11}$ Robert M. Light, ${ }^{3}$ \\ C. Roger Lynds, ${ }^{2}$ Earl J. O’Neil, JR., ${ }^{2}$ Edward J. Shaya, ${ }^{6}$ and James A. WestPhal ${ }^{12}$ \\ Received 1990 October 1; accepted 1990 November 19
}

\begin{abstract}
We have observed the nearby S0 galaxy NGC 7457 with the Planetary Camera of the Hubble Space Telescope. Spatial structure is observable at the diffraction-limited resolution of the $2.4 \mathrm{~m} \mathrm{HST}$ primary despite the effects of spherical aberration. The central distribution of starlight appears consistent with a $\gamma \approx-1.0$ power law for $r<600 \mathrm{pc}$, plus a possible additional stellar nucleus with $L \sim 1 \times 10^{7} L_{\odot}(V$ band). We find no evidence of a core to limits $r_{c}<0.052=3.4 \mathrm{pc}\left(H_{0}=80 \mathrm{~km} \mathrm{~s}^{-1} \mathrm{Mpc}^{-1}\right)$, in contrast to a priori estimates of $r_{c} \approx 30 \mathrm{pc}$ based on total galaxy luminosity. The center of NGC 7457 remains unresolved at $H S T$ resolution and has a central luminosity density $\rho_{0}>3 \times 10^{4} L_{\odot} \mathrm{pc}^{-3}(V$ band). This is now the second densest core known after M32. From the ground, NGC 7457 resembles any number of unresolved elliptical galaxies, which suggests that compact dense cores may be common. The images of NGC 7457 demonstrate that HST can still provide unique and astrophysically interesting information on the central structure of galaxies.
\end{abstract}

Subject headings: galaxies: nuclei — galaxies: photometry - galaxies: structure

\section{INTRODUCTION}

Our knowledge of the central structure of nearby galaxies is strongly limited by the effects of atmospheric seeing. Although many galaxies do show resolvable cores from the ground under excellent conditions, it was expected that only the Hubble Space Telescope (HST) could routinely provide data on the scale of the central few parsecs for nearby galaxies. Unfortunately, the strong spherical aberration present in the HST primary mirror severely compromises all programs requiring high-resolution imagery. Use of $H S T$ to investigate the central structure of galaxies now presents a difficult problem. While many nearby galaxies are bright enough in their cores to enable the use of deconvolution algorithms, which work best on high signal-to-noise ratio images, the interest here is on the quantitative distribution of light. Deconvolution algorithms must be faithful to the object light distribution, free of severe

\footnotetext{
${ }^{1}$ Based on observations with the NASA/ESA Hubble Space Telescope, obtained at the Space Telescope Science Institute, which is operated by AURA, Inc., under NASA contract NAS 5-26555.

${ }^{2}$ Kitt Peak National Observatory, National Optical Astronomy Observatories. Operated by the Association of Universities for Research in Astronomy, Inc., under cooperative agreement with the National Science Foundation. Postal address: National Optical Astronomy Observatories, P.O. Box 26732, Tucson, AZ 85726.

${ }^{3}$ UCO/Lick Observatories, Board of Studies in Astronomy and Astrophysics, University of California, Santa Cruz. Postal address: Lick Observatory, University of California, Santa Cruz, Santa Cruz, CA 95064.

${ }^{4}$ Lowell Observatory, 1400 Mars Hill Road, Flagstaff, AZ 86001.

5 Astronomy Department, University of Washington, FM-20, Seattle, WA 95195.

${ }^{6}$ Department of Physics and Astronomy, University of Maryland, College Park, MD 20742.

7 Space Telescope Science Institute, 3700 San Martin Drive, MD 21218.

8 Physics Department, Box 708, Princeton University, Princeton, NJ 08544.

9 Infrared Processing and Analysis Center, 100-22, California Institute of Technology, Pasadena, CA 91125.

${ }^{10}$ NASA/Goddard Space Flight Center, Laboratory for Astronomy and Solar Physics, Code 685, Greenbelt, MD 20771.

11 The Observatories, Carnegie Institution of Washington, 813 Santa Barbara Street, Pasadena, CA 91101.

${ }^{12}$ Division of Geological and Planetary Sciences, 170-25, California Institute of Technology, Pasadena, CA 91125.
}

artifacts or nonlinearity, and the relationship of the deconvolved image to both the object and the original image must be clear. The HST project recently completed an ad hoc Science Assessment program to evaluate use of the telescope in its present condition for the already planned research observations. Part of this program included images of NGC 7457, a nearby S0 galaxy with an unresolved core as observed from the ground. We present here a quick analysis of these images. We find that the $H S T$ can still produce images superior in resolution to those from the ground. NGC 7457 remains unresolved with HST, but we now find that any core most likely has $r_{c}<0$ ".052. The light distribution is consistent with a $\gamma=-1.0$ power law inward to the resolution limit, with a possible stellar nucleus with $L \sim 1 \times 10^{7} L_{\odot}(V$ band $)$. This result is of astrophysical interest. It represents the first observation outside the Local Group of a galaxy nucleus at this spatial resolution, and it suggests that such small, high surface brightness cores may be common.

\section{OBSERVATIONS}

NGC 7457 is a nearby S0 galaxy that is not a member of any obvious group. Its $V_{0}$ relative to the Local Group is $\sim 1000 \mathrm{~km}$ $\mathrm{s}^{-1}$, putting it at a distance of $12.5 \mathrm{Mpc}$ for $H_{0}=80 \mathrm{~km} \mathrm{~s}^{-1}$ $\mathrm{Mpc}^{-1}$. This gives the physical scale of $61 \mathrm{pc} \operatorname{arcsec}^{-1}$ or $2.6 \mathrm{pc}$ per Planetary Camera (PC) pixel; and $M_{B}=-19.22$, given $m_{B}=11.26(\mathrm{RC} 2)$. The bulge luminosity is -18.47 , for a bulgeto-disk ratio of 1.0 (Dressler \& Sandage 1983). High-resolution ground-based observations of NGC 7457 were obtained by Lauer (1985a). At the prevailing seeing, only an upper limit of $r_{c}<1$.53 for any core present could be measured. Partial deconvolution of the photometry refined this limit to $r_{c}<$ 1 ". 10 , or $67 \mathrm{pc}$. The average core radius at the luminosity of NCG 7457 as determined from the mean relations of Kormendy (1985a) and Lauer (1985b) for elliptical galaxies and S0 bulges is $r_{c} \approx 30 \mathrm{pc}$. The limits on the core size of NGC 7457 prior to this study were thus not particularly interesting, and this object was selected for the early science assessment program because it potentially had a core that could be resolved by $H S T$. 
$H S T$ observations of NGC 7457 were obtained with the PC or high-resolution mode of the Wide-Field/Planetary Camera (WFPC). Detailed description of the camera is presented in Griffiths (1989), and standard reduction procedures are presented by Lauer (1989). Briefly, the PC comprises four CCD cameras imaging a contiguous $66^{\prime \prime} \times 66^{\prime \prime}$ field of view. Each CCD camera has an $800 \times 800$ pixel format with 0 ".043 per pixel. Two $400 \mathrm{~s}$ integrations each were obtained with filters F555W and F785LP, which correspond roughly to the standard Johnson $V$ and $I$ bands. The two exposures in each set were compared to reject cosmic rays and were then added. The spacecraft was locked on guide stars during the exposures for each filter set, and the pictures using the F555W filter could be added directly with no offset. A 0 ".02 offset occurred between the two F785LP exposures, however, and it was necessary to shift one exposure to match the other. This was done with sinc-function interpolation; the pictures of the galaxy, while sharp, do not contain sufficient aliased power to present difficulties for this method. The total signal for each filter exceeds $10^{4}$ photons in the central pixel. At this scale and integration time, sky background is negligible.

At the time of the observations, the WFPC was still in an uncalibrated state prior to the application of the "UV flood." A combination of ground-test data and provisional on-orbit calibrations allowed most of the basic reduction steps outlined in Lauer (1989) to be completed. The main problems remaining are flat-fielding and quantum efficiency hysteresis (QEH). Onorbit flats have not been generated yet, so ground-test flats were used. These are of limited use and some CCD response variations remain in the images. The variation of the $C C D$ quantum efficiency with the illumination pattern $(\mathrm{QEH})$ prior to the flood is more problematic, and it is possible that the F555W images suffer from some nonlinearities at the few percent level (this effect is less important in the red).

The appearance of the reduced images is striking - the sharp nucleus stands out clearly from the surrounding galaxy (Fig. 1 [P1. L10]). It is immediately apparent that the center of NGC 7457 is extremely compact. The light distribution of NGC 7457 was measured with the high-resolution ellipse fitting software routines of Lauer (1985a). The observed $V$-band (F555W) and $I$-band (F785LP) profiles are presented in Figure 2. The normalization has been set by matching the outer parts of both profiles to the $R$-band profile of Lauer (1985a). The halfintensity radii or "apparent" cores of the $V$ - and $I$-band profiles are 0 .064 and 0.086 , respectively. This resolution is well in excess of any measurement that can be done from the ground and shows that even in its compromised state $H S T$ can still deliver useful information close to its diffraction limit. In fact, the rise of the $V$ profile above the $I$ profile interior to $r \approx 0$ ". 15 is most likely due to wavelength dependence of the size of the central diffraction spike of the PSFs rather than to a blue nucleus intrinsic to NGC 7457: the $V$ profile is $0.6 \mathrm{mag}$ brighter in the center than the $I$ profile, which differs negligibly from the difference of $0.5 \mathrm{mag}$ in the PSFs. Even at the present resolution, however, the galaxy nucleus must still be considered to be unresolved, and either profile's half-intensity radius is only an upper limit to any true core physically present in the galaxy. Although the half-intensity radii are twice as large as the half-intensity radii of the PSF, simulations conducted by several authors (e.g., Schweizer 1979; Bailey \& Sparks 1983) show that this is easy to explain as the simple blurring of an intrinsic power-law light distribution. Indeed, it is possible that the galaxy may not contain any core or central leveling-off of the light distribution at all.

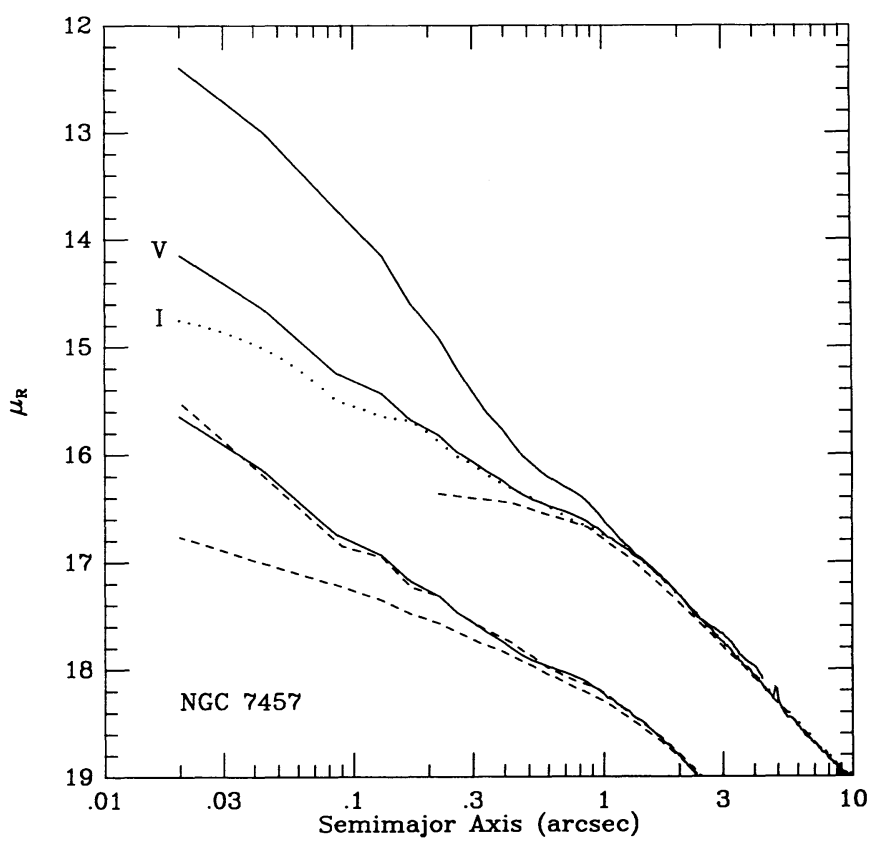

FIG. 2.-Surface brightness profiles of NGC 7457. The two central traces in the upper set of profiles are the $V$ and $I$ band brightness profiles of NGC 7457 (solid and dotted lines, respectively), scaled to Johnson $R$, and plotted as a function of isophote semimajor axis length. The divergence of the two profiles for $r<0$ ". 1 is most likely due to wavelength dependence of the size of the central PSF diffraction spike. $R$-band normalization is provided by the Lauer (1985a) ground-based profile, shown as a dashed line. The upper solid line is the deconvolved $V$-band profile after 20 iterations of Lucy's (1974) method. The lower set of profiles shows two models convolved with the PSF matched to the $V$-band profile as observed. The solid line is a repeat of the $V$-band profile (offset by $1.5 \mathrm{mag}$ ). The lower dashed line is the pure $\gamma=-1.0$ power law. The dashed line that closely matches the $V$-band profile is the same $\gamma=-1.0$ model but with a central $m_{V}=17.7$ point-source added.

While details on the diffraction scale are readily apparent in the NGC 7457 images, relating the light distribution in the nucleus to that of the rest of the galaxy is made problematic by the complex structure of the PSF. In Figure 2 it is seen that, while the central light distribution of NGC 7457 interior to 1 " appears to be a simple power law, the slope there is shallower than that at larger radii. Superficially, this looks like a feature or bend in the light profile, but simulations presented in Figure 3 show that this bend is an artifact induced by the unusual PSF of the aberrated mirror. An approximate PSF image for filter F487N is shown in Figure $1 .^{13}$ Briefly, the PSF contains a central spike with width close to the diffraction limit but containing only $15 \%$ of the power, with the remaining light scattered into a large halo that extends to $3^{\prime \prime}$ radius. This halo is complex, showing diffraction rings and radial "tendrils" caused by the secondary mirror and its spider, mirror pads on the primary, and the PC internal secondary and spider (see Burrows et al. 1991). The core of NGC 7457 is compact enough that these halo features in the PSF are clearly visible in the galaxy and greatly complicate measurement of the brightness profile - the bumps and wiggles in the observed profiles in Figure 2 are not due to noise, but to complex PSF structure.

The strong effect of the PSF on the light profile means that any quantitative results gleaned from the inner arcsecond of NGC 7457 must fully take the PSF into account out to large

${ }^{13}$ Unfortunately, errors in the $H S T$ scheduling system prevented execution of separate exposures that had been planned to observe PSF stars for the same filters and field location as used for the galaxy core. 


\section{PLATE L10}

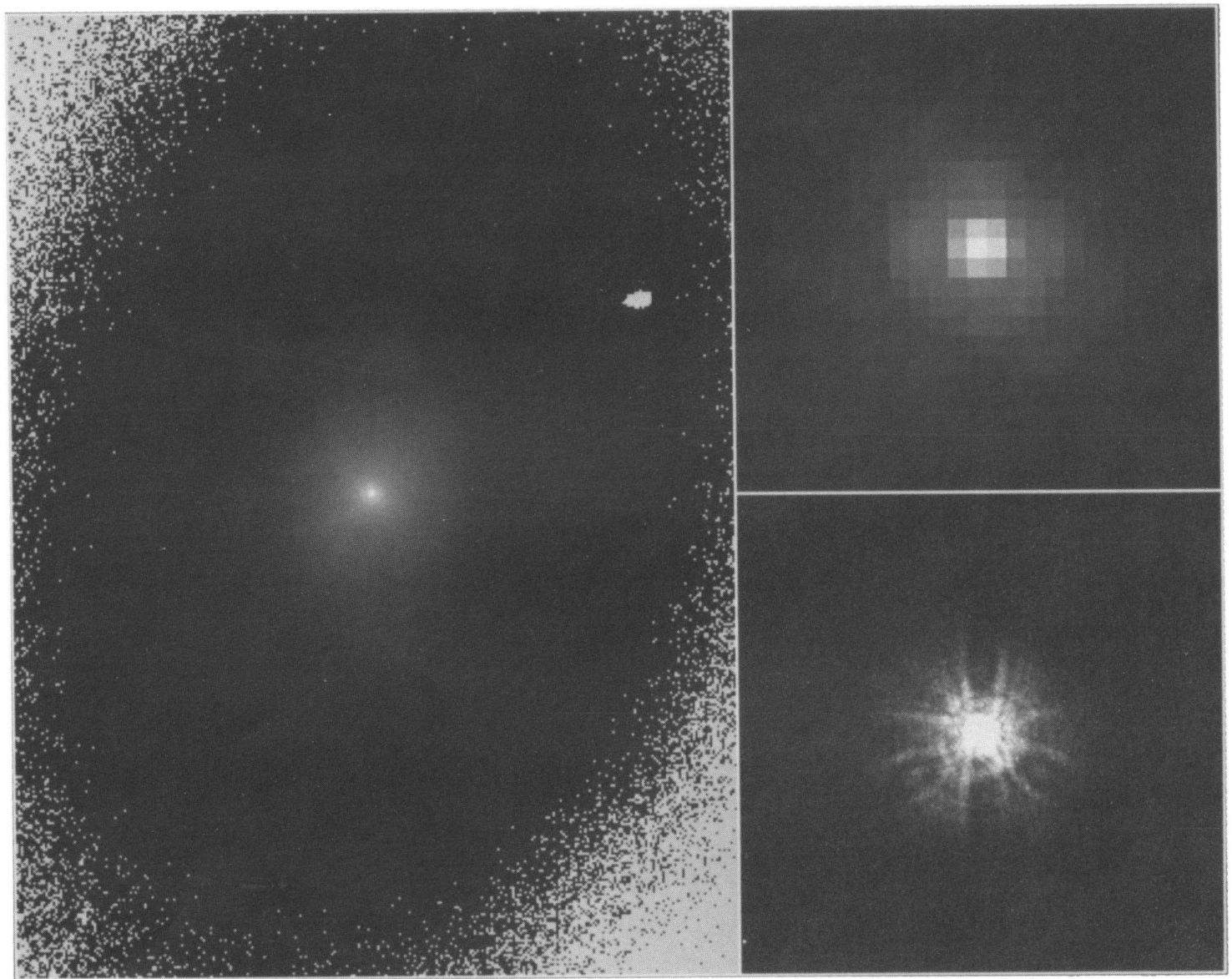

FIG. 1.-PC images of NGC 7457 and typical PSF. The large panel shows the central $400 \times 300$ pixels or $17 " .2 \times 12$ ".9 region of the F555W image centered on the nucleus of NGC 7457. The gray scale is logarithmic with the stretch set so that the outer isophote marks a decrease of 4 mag in surface brightness from the center. The compactness of the core is evident. The small panel to the upper right is an $8 \times$ magnified view of the core, now shown with a linear gray scale. The panel is only 25 pixels or $1^{\prime \prime} 08$ on a side, and it is immediately clear that $H S T$ is sensitive to structure in NGC 7457 close to its theoretical diffraction limit. The last panel shows a PC PSF taken in filter F487N. The stretch is set to show both the extent and complexity of the halo. This panel is 8".6 $\times 8$ ".6 and has the same magnification as the first panel.

LAUER et al. (see 369, L42) 


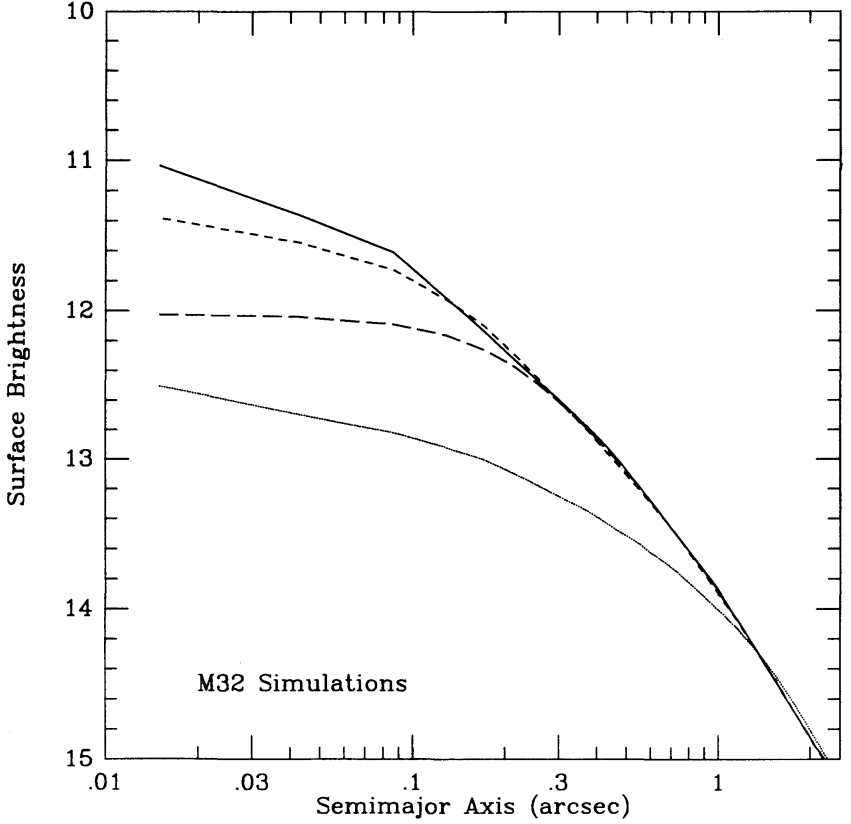

Fig. 3.-Simulated surface brightness profiles. The upper trace is a simulated image of M32 observed with a perfect HST. The input profile (not shown) is a $\gamma=-0.75$ power law for $r<0$. 4 , and $\gamma=-1.10$ for $r>0$. 4 . The bottom trace shows the same galaxy observed with $H S T$ with its optics as delivered on orbit. Spherical aberration causes a strong depression in the central brightness of the galaxy and a decrease in slope of the central brightness profile that extends to large radii; however, the power-law nature of the innermost portion of the profile is still evident. In contrast, the second profile from the bottom shows the galaxy as observed with a 0"33 FWHM Gaussian PSF. The profile is brighter in the center but also much flatter on scales smaller than the seeing. The next profile up shows the bottom profile after 20 iterations of Lucy (1974) deconvolution. Resolution close to the theoretically perfect HST has been recovered.

radii. Given the complex structure of the PSF, such analysis must be done on the full two-dimensional image; no shortcuts that exploit the symmetry of the galaxy or PSF will work here. To investigate this problem, we have conducted a number of experiments on simulated $H S T$ observations of galaxies using Lucy (1974) deconvolution to correct in part for the effects of the PSF. Lucy deconvolution appears to work well on galactic nuclei, as shown by the deconvolution simulations presented in Figure 3. Four profiles are shown in this figure, which gives simulated results for $\mathrm{M} 32$, a galaxy that now appears similar to NGC 7457. For this exercise, the M32 brightness profile was modeled as a power law that rises steeply into the nucleus at first and then turns into a $\gamma=-3 / 4$ cusp consistent with the presence of central massive black hole (Young 1977). The top profile shows M32 as observed with a perfect $H S T$, while the bottom shows it observed with $H S T$ as delivered. The bottom profile still shows the central cusp, but with the central surface brightness depressed by $\sim 1.5$ mag. The third profile down shows M32 as observed with 0".33 FWHM Gaussian seeing, comparable to the best that can be achieved from the ground. This profile is brighter in the center, but also flatter interior to the seeing radius, showing that $H S T$ even in its present condition can deliver information on spatial scales finer than can be achieved at the best ground-based sites. The second profile down shows a Lucy deconvolution of the bottom profile. The resolution here approaches the original HST specifications, and no obvious artifacts or nonlinearities are present. This simulation used only 20 Lucy iterations; in practice one can continue at least twice as long with high $\mathrm{S} / \mathrm{N}$ images and approach the diffraction-limited results even more closely. As used here, the major effect of the deconvolution is to suppress the strong halo of the PSF, with only slight sharpening of the central core.

Lucy deconvolution of the $V$-band profile of NGC 7457 is presented in Figure 2. As noted, no PSF observations could be obtained at the time of the NGC 7457 observations, but a $V$-band PSF close to the same CCD location as the NGC 7457 core was obtained about 1 month later in the course of another program. The OTA optics had been adjusted in the mean time, and the PSF is likely to have changed in the diffraction core, but probably has about the correct energy distribution at large radii, which is the most important aspect here. Artifacts are seen in the deconvolution, but without a PSF known to be perfectly valid this should not be taken as a negative reflection on Lucy deconvolution, itself; and even with these problems, the deconvolved profile serves as a useful loose constraint on the true structure of the galaxy.

In superficial detail, the deconvolved profile suggests that a lower bound on the light distribution interior to the inner 1 " is a simple inward continuation of the $\gamma \approx-1.0$ power-law brightness distribution seen at larger radii. The observed profile interior to $1^{\prime \prime}$ is a simple power law but with shallower slope than in the outer parts. We know from simulations that this decrease in slope is due to the PSF halo. We expect the deconvolved profile to be steeper here, and this is the case. Indeed, simulations indicate that the central surface brightness should increase in the center by at least $1.5 \mathrm{mag}$, which agrees well with the observed brightening of 2.0 mag. Any core present is likely to be smaller than the deconvolved profile half-intensity radius, which implies $r_{c}<0$ ".052, or $r_{c}<3.2 \mathrm{pc}$.

While a power law is the simplest model, however, we note that several nearby galaxies, such as M31, have compact and distinct nuclear star clusters embedded in a larger resolved core, and a nuclear star cluster may be present in NGC 7457, as well. The second set of profiles shown in Figure 2 show the results of matching PSF-convolved models to the data to verify the deconvolution. A simple $\gamma=-1.0$ power law convolved with PSF in fact predicts less light in the center than seen and has a half-intensity radius of 0 .'21 that is not as sharp as the observed 0.064 value. Only when we add a central point source with $L \sim 1 \times 10^{7} L_{\odot}\left(m_{V}=17.7\right)$ to the pure power law do we get a good match to the $V$-band profile as observed. The conclusion that any core present is unresolved appears robust, however. We tried a number of models with well-defined cores, but with even stronger central point sources in an attempt to match the total light in the center. Even with $r_{c}$ as small as 0 ".075, however, it was not possible to get as good a match to the data as a point source added to a $\gamma=-1.0$ model. This picture is not in conflict with the deconvolved profile. Only 20 Lucy iterations were used in the deconvolution experiments, and the width of the deconvolved PSF is significant. As is evident in Figure 3, the deconvolved profile at this stage still does not match even the diffraction-limited perfect $H S T$. Likewise, the true profile of NGC 7457 will be sharper still than the deconvolved profile shown in Figure 2, and a nucleus might be recovered by pushing Lucy deconvolution further on images with higher signal-to-noise ratio and better knowledge of the PSF than we had here.

\section{DISCUSSION}

NGC 7457 from the ground is no different than any number of other nearby unresolved galaxies, suggesting that many other galaxies may be equally compact. While Lauer (1985b) 
and Kormendy (1985a) find that in general core size correlates with galaxy luminosity, Lauer (1985b) also shows that central luminosity density can range over nearly two orders of magnitude at any total luminosity. The present observations of NGC 7457 emphasize the concern that the real range in central density at any galaxy luminosity remains undetermined. Previous limits on the core of NGC 7457 were on the large side of the mean core-luminosity relation. The present results show that any core present in NGC 7457 is now over an order of magnitude more compact than the mean predicted value.

NGC 7457 is unresolved with $r_{c}<3.2 \mathrm{pc}$, placing it second only to M32 with $r_{c}<0.9$ pc (Lugger et al. 1991) in having the smallest core known. The light profile also resembles that of M32, which is well described by a $\gamma=-1.2$ power law for $r>3 \mathrm{pc}$. The central luminosity density of NGC 7457 is $\rho_{0}>3$ $\times 10^{4} L_{\odot} \mathrm{pc}^{-3}$ ( $V$ band), again second only to M32, which, with $\rho_{0}>5 \times 10^{5} L_{\odot} \mathrm{pc}^{-3}$, is the densest nucleus known. NGC 7457 shows other parallels with M32: the central velocity dispersion is roughly $80 \mathrm{~km} \mathrm{~s}^{-1}$ (Whitemore, McElroy, \& Tonry 1985; Dalle Ore et al. 1991), and the nuclear metal line strength is low for an E/S0 stellar population (Faber et al. 1991). M32 is an excellent nuclear black hole candidate (Tonry 1987; Dressler \& Richstone 1988), suggesting that NGC 7457 may be an interesting target for high spatial resolution spectroscopy as well.

The probable power-law light distribution of NGC 7457 is also interesting. Kormendy (1985b) and Lauer (1985b) have both shown that the central regions of elliptical galaxies are poorly described by the traditional King (1966) or de Vaucouleurs (1958) models. Ellipticals with unresolved cores often have more light in the center than can be accounted for by de Vaucouleurs models fitted to their envelopes (Lauer 1985b)- indeed, many have power-law profiles similar to NGC 7457 beyond $r>1$ ". The presumption has been that cores would be observed in these galaxies with improved resolution, but the examples of M32 and now NGC 7457 show that such assumptions may be unwarranted. An ongoing problem has been understanding how a massive black hole could form in a galactic nucleus and then become visible as an AGN. Theoretical arguments suggest that black holes and power-law profiles may be intimately related, either by the formation of a stellar density cusp in response to the adiabatic growth of a black hole (Young 1977) or, turning the problem around, by the formation of a black hole via stellar collisions in a preexisting cusp. Either way, the discovery of another galaxy like M32 that exhibits a power law down to very small radii is noteworthy.

The final conclusion concerns the utility of this type of research with Space Telescope in its compromised condition. To obtain useful information on galaxy cores requires longer exposure times (by at least a factor of 4 for the most compact objects) and exquisite care paid to the accuracy of the PSF. The profile can be analyzed only with deconvolution or by PSF-convolved model fitting. We have tried both approaches here and in the former case find that Lucy (1974) deconvolution can recover useful quantitative information on the light distribution. Despite these caveats, the scientific rewards are large, and imaging of galactic nuclei belongs to the class of high surface brightness problems for which the Hubble Space Telescope retains much of its original capability.

This research was conducted by the WFPC Investigation Definition Team operating under partial support from NASA contract NAS 5-25421.

\section{REFERENCES}

Bailey, M. E., \& Sparks, W. B. 1983, MNRAS, 204, 53P

Burrows, C., Holtzman, J. A., Faber, S. M., Bely, P., Husan, H., Lynds, C. R., \& Schroeder, D. 1991, ApJ, 369, L21

Dalle, Ore, C., Faber, S. M., Burstein, D., Gonzalez, J., \& Stoughton, R. 1991, ApJ, 366, 38

de Vaucouleurs, G. 1958, Handbuch der Physik, 53, 275

Dressler, A., \& Richstone, D. O. 1988, ApJ, 324, 701

Dressler, A., \& Sandage, A. 1983, ApJ, 265, 664

Faber, S. M., Worthey, G., Gonzalez, J., Dalle Ore, C., \& Burstein, D. 1991, in

preparation
Griffiths, R. 1989, Wide Field and Planetary Camera Instrument Handbook (STScI publication)

King, I. R. 1966, AJ, 71, 64
Kormendy, J. 1985a, ApJ, 295, 73

. 1985b, ApJ, 292, L64

Lauer, T. R. 1985a, ApJS, 57, 473

1985b, ApJ 292,104

1989, PASP, 101, 445

Lugger, P. M., Cohn, H. N., Cederbloom, S. E., Lauer, T. R., Grindlay, J. E., Bailyn, C. D., \& McClure, R. D. 1991, in preparation

Lucy, L. B. 1974, AJ, 79, 745

Schweizer, F. 1979, ApJ, 233, 23

Tonry, J. L. 1987, ApJ, 322, 932

Whitmore, B. C., McElroy, D. B., \& Tonry, J. L. 1985, ApJS, 59, 1

Young, P. J. 1977, ApJ, 217, 287 